\title{
Rentenpolitik zwischen Rollenbildern und Respekt: Verletzen Grundrente \& Co. die Grundprinzipien der Gesetzlichen Rentenversicherung - oder helfen sie vielmehr, ihr Sicherungsversprechen einzulösen?
}

\author{
Ute Klammer
}

\section{Einleitung}

Die gesetzliche Alterssicherung mit ihrer weit über 100jährigen Geschichte steht seit Jahren von unterschiedlichen Seiten unter Druck: Plädieren die einen für weitere Reduzierungen der Leistungshöhe mit Blick auf das Ausgabenvolumen, die steigenden Kosten durch die Alterung der Gesellschaft und insbesondere die hiermit verbundenen Belastungen des Faktors Arbeit durch Sozialbeiträge, wird von anderer Seite auf die problematischen Folgen der seit 2001 eingeleiteten Rentenniveauabsenkungen in der GRV aufmerksam gemacht. Der im Gegenzug postulierte, freiwillige Aufbau der betrieblichen und privaten Vorsorge als „zweiter und dritter Säule“ der Alterssicherung ist deutlich hinter den Erwartungen zurückgeblieben und erweist sich nicht als geeignet, die Ungleichheit von Alterseinkommen zu vermindern und armutsgefährdete Personengruppen vor Armut im Alter zu schützen. Dabei trifft das Risiko, keine armutsvermeidenden Rentenansprüche ${ }^{1}$ aufbauen zu können, nicht nur Personen, deren Biografie durch besondere „Wechselfälle des Lebens“ wie Verschuldung, Scheidung oder Fluchtmigration geprägt ist. Vielmehr gelingt es auch Menschen, deren Erwerbs- und Familienleben entlang gängiger gesellschaftlicher und sogar politisch geförderter Normvorstellungen verlaufen ist, oft nicht (mehr), genug Rentenansprüche aufzubauen, um im Alter vor Armut und Angewiesenheit auf Grundsicherung geschützt zu sein. Gründe dafür sind beispielsweise, dass sie Arbeit zu Niedriglöhnen oder in Teilzeitbeschäftigung oder längere Phasen vorwiegend Familienarbeit ausgeübt haben. So stellt sich immer dringlicher die Frage: Wie kann im Rentensystem ein auskömmliches Leben im Alter auch für diejenigen sichergestellt werden, die

1 Hierunter werden hier Rentenansprüche oberhalb des Grundsicherungsniveaus (als gesellschaftlich definierter Schwelle „bekämpfter Armut“) verstanden. 
angesichts der (arbeitsmarkt)politischen und gesellschaftlichen Entwicklungen aufgrund niedriger Erwerbseinkommen, aber auch familienbedingter Erwerbseinschränkungen momentan keine armutsvermeidenden Rentenansprüche erzielen (können)?

Der Beitrag beleuchtet diese Fragestellung in mehreren Schritten. Zunächst werden Fakten zur Entwicklung niedriger Alterssicherungsansprüche betrachtet. Anschließend werden Studienergebnisse zur Wahrnehmung von sozialer Ungleichheit und Armutsrisiken reflektiert und es wird gefragt, welche Erwartungen und Gerechtigkeitsvorstellungen die Menschen in Deutschland in Bezug auf den Sozialstaat und insbesondere die Alterssicherung haben. Hieran schließt sich eine Diskussion ausgewählter Handlungsansätze an, die dazu beitragen können, die Lebensleistung von Personen zu honorieren, die sich im Alter mit geringen Rentenansprüchen oder finanzieller Abhängigkeit konfrontiert sehen, obwohl sie gesellschaftlich akzeptierten und sogar staatlich unterstützten Lebens- und Erwerbsmodellen gefolgt sind. Als staatliches Rentensystem der ersten Säule, so die Argumentation, steht die GRV vor der Aufgabe, auch für diese Gruppe ein „Sicherungsversprechen“ einzulösen, wenn sie nicht Gefahr laufen will, weiter an Akzeptanz und Vertrauen zu verlieren.

\section{Fakten und Prognosen: Armut und finanzielle Abhängigkeit im Alter}

Zurecht wird immer wieder darauf verwiesen, dass ältere Menschen in Deutschland - auch dank des existierenden Alterssicherungssystems - gegenwärtig (zumindest nach Daten des Mikrozensus) keinem vermehrten Armutsrisiko ausgesetzt sind. Die Quote der älteren Grundsicherungsempfänger liegt deutlich unter denjenigen jüngerer Altersgruppen und besonderer Problemgruppen wie alleinerziehender Mütter. Die Quote der Grundsicherungsbezieher*innen im Rentenalter ist jedoch, wie Abb. 1 verdeutlicht, im Zeitverlauf immer weiter (wenn auch auf niedrigem Niveau) gestiegen. 2018 bezogen 3,1\% aller Personen im Rentenalter 3 , 2\% der Frauen, 2,9\% der Männer) Grundsicherungsleistungen nach SGB XII. Dabei liegt die Bezugsquote bei älteren Frauen seit der Einführung dieser Leistung höher als diejenige der Männer, allerdings haben sich die Inanspruchnahmequoten in den vergangenen Jahren durch einen überproportionalen Anstieg älterer Männer im SGB XII-Leistungsbezug angeglichen. Absolut ist die Zahl der Empfänger*innen von Grundsicherung im Alter und bei Erwerbsminderung seit der Einführung kontinuierlich angestie- 
gen (von knapp 439.000 in 2003 auf knapp 1,06 Mio. 2017), wobei etwa die Hälfte (2017: 515.000 Personen) die Regelaltersgrenze überschritten hat.

Abbildung 1: Empfängerquoten von Grundsicherung im Alter und bei Erwerbsminderung 2003-2018 in \% der jeweiligen Bevölkerungsgruppe, am Jahresende

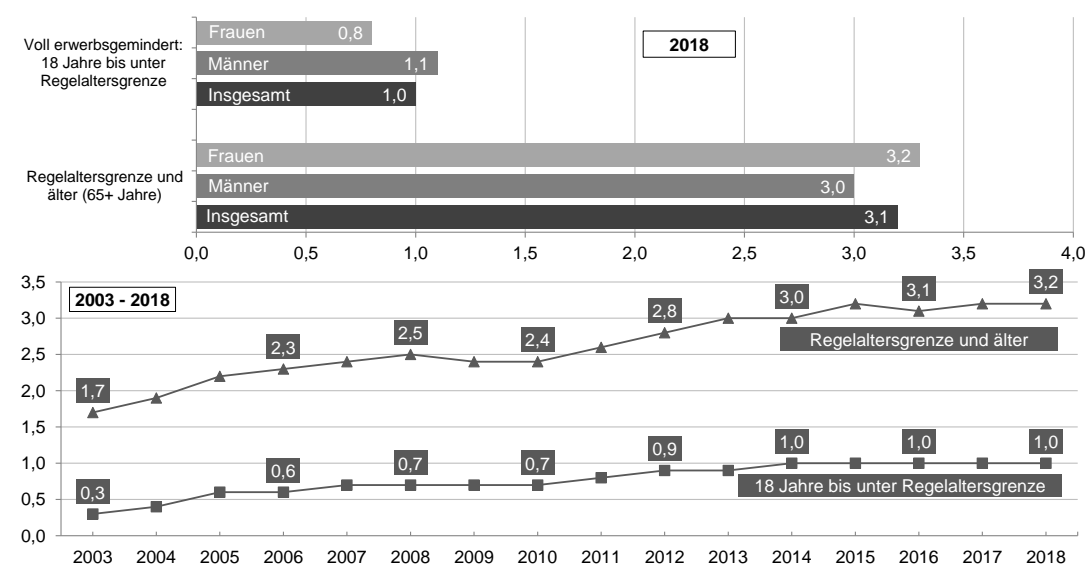

Quelle: Sozialpolitik-aktuell.de (2020) auf Grundlage von Daten des statistischen Bundesamtes. Anmerkungen: Bis 2014 zum Stichtag 31.12., ab 2015 für Dezember des jeweiligen Jahres. Bis 2011 lag die Altersgrenze bei 65 Jahren; ab 2012 wird die Altersgrenze bis zum Jahr 2031 auf 67 Jahre angehoben; 2018 betrug die Altersgrenze 65 Jahre und 7 Monate.

Bei der Bewertung der auf den ersten Blick nicht dramatisch erscheinenden Zahlen ist jedoch dreierlei zu berücksichtigen.

1. Zum einen ist von einer hohen Nichtinanspruchnahmequote bei älteren Menschen auszugehen; das DIW schätzt den Anteil auf $60 \%$ der Berechtigten (Buslei et al. 2019). Gründe können z.B. Unwissenheit über eigene Ansprüche sein oder fehlender Kompetenzen, diese geltend zu machen, Scham oder die (wenn auch oft unbegründete) Furcht davor, dass die eigenen Kinder überprüft und in Anspruch genommen werden könnten. Insofern dürfte die Armutsgefährdung im Alter deutlich höher liegen, als es die Inanspruchnahme von Grundsicherung abbildet.

2. Vor allem Frauen sind oft trotz niedriger eigenständiger Renten aufgrund der Berechnung des Grundsicherungsanspruchs auf Basis der Be- 
darfsgemeinschaft angesichts des Einkommens des (Ehe-)Mannes nicht anspruchsberechtigt. In einer ehelichen Zugewinngemeinschaft - dem Regelmodell der Ehe in Deutschland - bedeutet dies aber nicht, dass ein Anrecht auf die Verfügung über die Hälfte des Haushaltseinkommens besteht. Anders sähe dies im Güterstand der „Errungenschaftsgemeinschaft" aus, der u.a. in Belgien, Frankreich, Italien, Luxemburg und Portugal gesetzlicher Güterstand ist. Witwen sind in Deutschland zwar häufig durch Hinterbliebenenrenten gut gestellt und haben daher keinen Grundsicherungsanspruch. Jedoch stellen Witwenrenten „Ansprüche zweiter Klasse“ dar, die zum einen kein Abbild der eigenen Lebensleistung sind, zum anderen Handlungsoptionen für das weitere Leben, z.B. in Bezug auf eine neue Heirat, einschränken (vgl. Bundesregierung 2011, 2017).

3. Schließlich muss davon ausgegangen werden, dass der Anteil der Personen, die einen nicht armutsvermeidenden Rentenanspruch in der GRV erwerben, durch das Zusammenwirken verschiedener Faktoren wie der Absenkung des Rentenniveaus, langen Phasen von Erwerbstätigkeit mit Niedriglöhnen und/oder Teilzeitarbeit, veränderten Familienstrukturen, aber auch vermehrter Zuwanderung künftig steigen wird (Kumpmann et al. 2010). ${ }^{2}$ Die Studie Altersvorsorge in Deutschland (AVID) macht insbesondere vier zentrale Risikogruppen aus: Erwerbsgeminderte, langjährige Geringverdiener, Langzeitarbeitslose und Soloselbstständige; hinzu kommen Zugewanderte, denen es oft aufgrund der nur noch verbleibenden Zeit bis zum Rentenalter oder aber durch eine unzureichende Integration in den deutschen Arbeitsmarkt nicht mehr gelingt, armutsvermeidende Alterssicherungsansprüche aufzubauen (Brettschneider/Klammer 2016).

Die Absenkung des Rentenniveaus in der GRV erhöht die Hürde, durch eigene GRV-Rentenansprüche den allen bei Bedürftigkeit zustehenden Anspruch auf Grundsicherung zu übertreffen (s. Bäcker in diesem Band): Benötigte ein Durchschnittsverdiener im Jahr 2009 26,2 Versicherungsjahre, um einen Rentenanspruch auf Höhe des Grundsicherungsniveaus (inklusive Miet- und Heizkosten) zu erwirtschaften, wird er im Jahr 2030 ceteris paribus voraussichtlich 32,0 Erwerbs- und Versicherungsjahre dafür benötigen. Für eine Person, die aufgrund niedriger Stundenlöhne oder Teilzeit-

2 Dem stehen gegenläufige Entwicklungen wie die vermehrte Erwerbstätigkeit von Frauen, gesunkene Arbeitslosigkeit und die Einführung von Mindestlöhnen gegenüber, die in der Summe jedoch die Tendenz steigender Armutsgefährdung im Alter wohl nicht umkehren können. 
arbeit im Durchschnitt nur halb so viel verdient, waren schon 2009 utopische 52,4 Erwerbs- und Versicherungsjahre erforderlich; hier wird die Lücke zwischen GRV-Rentenanspruch und der Höhe der Grundsicherungsleistungen weiter wachsen und ein armutsvermeidender GRV-Rentenanspruch ist auch bei „lebenslanger“ Erwerbstätigkeit niemals zu erreichen. Vor diesem Hintergrund liegt die Frage auf der Hand, ob sich für die Betroffenen Einzahlungen in das GRV-Rentensystem überhaupt noch lohnen und ob die Rentenversicherung noch in der Lage ist, Altersarmut in der Breite zu verhindern.

\section{Wie nehmen Menschen Einkommensungleichheit und Armutsrisiken wahr und was erwarten sie vom Sozialstaat?}

Im Grundgesetz der Bundesrepublik Deutschland bildet die Sozialstaatlichkeit die Grundlage der freiheitlich-demokratischen Grundordnung (Art. 20 Abs. 1 GG): „Die Bundesrepublik Deutschland ist ein demokratischer und sozialer Bundesstaat“. Die Staatsziele Menschenwürde, Menschenrechte und Sozialstaatlichkeit genießen im Grundgesetz der Bundesrepublik Deutschland die Ewigkeitsgarantie des Art. 79 Abs. 3 GG. So besteht die Verpflichtung des Staates, das Existenzminimum zu gewährleisten (Art. 1 I GG i.V.m. dem Sozialstaatsprinzip aus Art. 20 I, 28 I GG). Kern der Auslegung des deutschen Sozialstaatsgebots war stets auch die Absicherung gegen die „großen Lebensrisiken“, so wie sie im Rahmen der fünf Sozialversicherungszweige deutlich wird. Wie aber nehmen die Menschen in Deutschland den Sozialstaat und seine Entwicklungen wahr? Eine Auswertung vorliegender Studien zu den Erwartungen der Menschen an den Sozialstaat (Brussig/Klammer/Lange 2019) macht deutlich: Es gibt eine überwältigende Zustimmung für die Zuständigkeit des Staates für die soziale Sicherung, die in der Bevölkerung der Bundesrepublik seit der Wiedervereinigung (1990) durchgängig bei mindestens $85 \%$ lag. Die Auffassung, dass der Staat zuständig dafür ist, grundlegende arbeitsmarktbezogene Risiken abzusichern, ist besonders hoch bezogen auf das Risiko, aufgrund eines hohen Alters oder aufgrund von Krankheit nicht mehr arbeiten zu können. Demoskopische Umfragen zeigen allerdings ein widersprüchliches Bild: Mehrheitlich werden einerseits Steuer- und Beitragserhöhungen wie auch eine höhere Staatsverschuldung abgelehnt, zugleich aber auch Kürzungen sozialstaatlicher Leistungen. Wenn sich auch über die 1990er Jahre und die Jahre bis 2010 eine etwas rückläufige Unterstützung für den Sozialstaat feststellen lässt, so lag die Unterstützung doch stets bei mindestens $80 \%$ der westdeutschen Bevölkerung. In Ostdeutsch- 
land war sie durchgängig noch höher. Seit etwa 2012 steigt die Unterstützung für den Sozialstaat erneut an: In der westdeutschen Bevölkerung hat die Zustimmung mit ca. $90 \%$ inzwischen wieder das Niveau vom Anfang der 1990er Jahre erreicht und deckt sich nun etwa mit den Zustimmungsraten in der ostdeutschen Bevölkerung. Offensichtlich wird der Sozialstaat angesichts der wahrgenommenen Ungleichheit als wichtige Unterstützung angesehen.

Aktuelle Untersuchungen dazu, was die Menschen im Sozialstaat als "gerecht" empfinden, haben Wissenschaftler"innen des DIW vorgelegt (Eisnecker et al. 2018). Anders als die zuweilen große mediale Aufmerksamkeit für Vorschläge eines „bedingungslosen Grundeinkommens“ vermuten lassen würde, gibt es über die Parteigrenzen hinweg eine hohe $\mathrm{Zu}$ stimmung zu Verteilungsprinzipien, die dem (Vor-)Leistungsprinzip folgen. Zugleich gibt es aber auch eine hohe Zustimmung zum Bedarfsprinzip: Wer bedürftig ist, dem soll zumindest ein bescheidener Lebensstandard zugesichert werden. Die Befürwortung des Bedarfs- und des Leistungsprinzips ist in Deutschland sogar stärker ausgeprägt als in anderen europäischen Ländern (Adriaans et al. 2019). Hier zeigt sich die Erwartung, dass der Staat sowobl für eine verlässliche Mindestsicherung Sorge trägt, als auch die Organisation (vor-)leistungsbezogener Versicherungssysteme übernimmt, die helfen, den Lebensstandard von Versicherten bei Eintreten der großen Risiken wie Arbeitslosigkeit und Alter zu sichern. Von der Regierung werden Maßnahmen zur Reduzierung von Einkommensungleichheit erwartet. So zeigte sich in einem Survey von Götte (2015), dass $44 \%$ der Befragten in Deutschland einen Anstieg der Armut wahrnehmen. Zwei von drei Befragten sehen ein Risiko der Altersarmut; $50 \%$ glauben, den Armen werde in Deutschland genug abverlangt; zwei von drei Befragten meinen, Reiche sollen mehr abgeben. Im Statista-Dossier „Armut in Deutschland“ (2018) referierte Daten mehrerer Befragungen untermauern, dass ein Großteil der Bevölkerung erhebliche Ungerechtigkeiten bezüglich Armut und Ungleichverteilung von Einkommen sieht und unzufrieden mit den politischen Bemühungen hinsichtlich dieser Aspekte ist. Nur $39 \%$ fühlten sich für das Rentenalter genügend abgesichert. Nur 28\% der Befragten trauten der Bundesregierung zu, Altersarmut zu verhindern. Zugleich sahen die Befragten klar die Regierung in der Pflicht, Maßnahmen zu ergreifen, um die Einkommensunterschiede zu reduzieren (Statista 2018). 


\section{Was ist zu tun? Ausgewäblte Handlungsansätze}

Bei der Frage, wie die Risiken unzureichender Alterseinkünfte und finanzieller Abhängigkeit im Alter angegangen werden können, ist zu berücksichtigen, dass Altersarmut immer das Resultat einer individuellen Risikokombination und an verschiedenen Knotenpunkten in Leben eingetretener Ereignisse ist. Dies können neben Charakteristika der Erwerbsbiografie auch Merkmale der Familienbiografie, der Gesundheitsbiografie, des Bildungsverlaufs, von Einwanderung, mangelnder Vorsorge oder sonstige problematische Elemente der Biografie wie Verschuldung, Insolvenz oder Sucht sein (Tab. 1).

\section{Tabelle 1: Armut und unzureichende Absicherung im Alter-} Risiken im Lebenslauf

\begin{tabular}{|c|c|c|}
\hline Erwerbsbiografie & Familienbiografie & Gesundheitsbiografie \\
\hline $\begin{array}{ll}\text { - } & \text { Langzeitarbeitslosigkeit } \\
\text { - } & \text { langjähriger Niedrigver- } \\
& \text { dienst } \\
\text { - } & \text { langjährige geringfügige Be- } \\
& \text { schäftigung } \\
\text { - } \quad \text { nicht versicherungspflichtige } & \text { Selbstständigkeit } \\
\text { - } & \text { Schattenwirtschaft }\end{array}$ & $\begin{array}{l}\text { - } \begin{array}{l}\text { kindbedingte Unterbre- } \\
\text { chungen }\end{array} \\
\text { - } \quad \text { Angehörigenpflege } \\
\text { - Trennung/Scheidung/ } \\
\text { Verwitwung }\end{array}$ & $\begin{array}{ll}\text { - } & \text { Erwerbsminderung } \\
\text { - } & \text { Behinderung } \\
\text { - } & \text { sonstige Einschränkungen }\end{array}$ \\
\hline
\end{tabular}

\begin{tabular}{|c|c|c|}
\hline Bildungsbiografie & Migrationsbiografie & Vorsorgebiografie \\
\hline $\begin{array}{l}\text { - } \quad \text { fehlender Schulabschluss } \\
\text { - } \\
\text { - } \\
\text { - } \\
\text { fusbruchlende Weiterbildung }\end{array}$ & $\begin{array}{ll}\text { - } & \text { später Zuzug } \\
\text { - } & \text { ursprüngliche Rückkehr- } \\
\text { absicht } \\
\text { - } \quad \text { aufenthaltsrechtliche } \\
\text { Probleme } \\
\text { - } \begin{array}{l}\text { allgemeine Integrations- } \\
\text { probleme }\end{array}\end{array}$ & $\begin{array}{l}\text { - fehlender Zugang zu be- } \\
\text { trieblichen/privaten } \\
\text { Vorsorge } \\
\text { mangelnde Vorsorge- } \\
\text { fähigkeit } \\
\text { - mangelnde Vorsorge- } \\
\text { bereitschaft } \\
\text { gescheiterte Vorsorge- } \\
\text { strategie }\end{array}$ \\
\hline
\end{tabular}

Sonstige Elemente der Biografie

- Verschuldung, Insolvenz

- Kriminalität, Sucht, Obdachlosigkeit

- (Selbst-)Exklusion/Devianz, „Schicksalsschläge“

Quelle: Angelehnt an Brettschneider/Klammer (2016, S. 54).

Entsprechend bedürfen unterschiedliche Ursachen teilweise unterschiedlicher Antworten. Nicht alle Wechselfälle des Lebens können innerhalb des Systems der gesetzlichen Alterssicherung gelöst werden. Die folgenden Ausführungen konzentrieren sich daher auf den Ausschnitt derjenigen, die sich mit nicht armutsvermeidenden Rentenansprüchen konfrontiert se- 
hen, obwohl sie als langjährig rentenversicherte Erwerbstätige oder als Sorgearbeit Leistende ein gesellschaftlich anerkanntes, den Normen und Erwartungen entsprechendes Leben geführt haben. Im Zentrum steht die Frage, ob das Sicherungsversprechen der Rentenversicherung nicht (zumindest) für diese Fälle Ausgleichsmechanismen innerhalb der Sozialversicherung erforderlich macht.

\subsection{Wie kann die „Lebensleistung“ von Frauen besser abgebildet werden?}

Dass das stark erwerbszentrierte deutsche Alterssicherungssystem aufgrund der Orientierung an der (zumindest früher geltenden) männlichen Norm dauerhafter Vollzeiterwerbstätigkeit Frauenbiografien nur unzureichend gerecht wird, ist seit Jahrzehnten bekannt (vgl. z.B. Klammer 2016) und dennoch immer noch ein Befund, der bei der Betrachtung zukünftiger Reformoptionen Beachtung verdient. Da die abgeleiteten Hinterbliebenenrenten zwar nach wie vor großzügig sind (und damit möglicherweise zur Fortschreibung traditioneller Geschlechterrollenmodelle beitragen), gleichzeitig aber aufgrund ihres besonderen Charakters als Zwitter zwischen Versicherungs- und Fürsorgeleistung keinen adäquaten „Ersatz“ für eigenständige Ansprüche darstellen, muss das Augenmerk auf den Letztgenannten liegen. Woran mangelt es also Frauen vor dem Hintergrund der Folie des männlichen Erwerbslebensverlaufs, der dem „Eckrentner“ der GRV zugrunde liegt?

Tabelle 2: Eigenständige GRV-Altersrentenansprüche von Männern und Frauen in Deutschland (Rentenbestand am 31.12.2018) - Anteile des Zeitund des Einkommensfaktors an der geschlechtsspezifischen Rentenlücke

\begin{tabular}{lrrrrrr}
$\begin{array}{l}\text { Landes- } \\
\text { teil }\end{array}$ & \multicolumn{2}{c}{$\begin{array}{l}\text { Monatsrente in } \\
\text { Euro }\end{array}$} & \multicolumn{2}{c}{$\begin{array}{l}\text { Durchschnittliche } \\
\text { Versicherungszeit } \\
\text { in Jahren* }\end{array}$} & \multicolumn{2}{c}{$\begin{array}{l}\text { Durchschnittliche } \\
\text { Entgeltpunkte pro }\end{array}$} \\
& Männer & Frauen & Männer & Frauen & Männer & Frauen \\
\hline Gesamt & 1.223 & 701 & 41,53 & 29,92 & 1,04 & 0,75 \\
West & 1.224 & 636 & 40,53 & 26,92 & 1,05 & 0,73 \\
Ost & 1.221 & 942 & 44,75 & 41,16 & 1,01 & 0,82 \\
\hline
\end{tabular}

Anmerkung: * Beitragszeiten und beitragsfreie Zeiten bei Rentenberechnung nach Sozialgesetzbuch (SGB VI). Quelle: BMAS (2019), Rentenversicherungsbericht 2019 auf der Basis der Statistik der GRV Bund, S. 19; eigene Zusammenstellung. 
Wie Tab. 2 verdeutlicht, schlagen bei Rentnerinnen im Westen sowohl fehlende Versicherungszeiten (Erwerbsunterbrechungen) zu Buche wie auch unterdurchschnittliche Löhne (Gender Pay Gap). Bei heutigen Rentnerinnen im Osten mangelt es kaum an der Dauer der Versicherungszeiten (Zeitfaktor), während aber auch hier im Vergleich zu Männern eine deutliche Diskrepanz in der Höhe des Erwerbseinkommens (Entgeltfaktor) zu verzeichnen ist.

Zwar wird sich die bisher zu konstatierende negative Korrelation zwischen Kinderzahl und Rentenanspruch bei (westdeutschen) Müttern durch die gestiegene Erwerbstätigkeit dieser Gruppe, aber auch die verbesserte Anrechnung von Kindererziehungszeiten in der Rente, die auch in anderen europäischen Ländern zu verzeichnen war (Blank/Blum 2017), künftig abschwächen. Wenn es darum geht, der „Lebensleistung“ von Frauen mit niedrigen eigenständigen Rentenansprüchen Anerkennung zukommen zu lassen, sind jedoch weitere Maßnahmen angezeigt. Aus den Vorschlägen hierzu, die im Rahmen des 1. und 2. Gleichstellungsberichts (Sachverständigengutachten in: Bundesregierung 2011, 2017) entwickelt wurden, sollen im Folgenden zwei näher ausgeführt werden: Eine Verbesserung der Anrechnung von Pflegezeiten sowie die Einführung eines Anwartschaftssplittings für (Ehe-)Paare.

\subsubsection{Verbesserung der Anrechnung von Pflegezeiten}

Bis heute zeigt sich im Rentenrecht eine deutliche Ungleichbehandlung von Sorgearbeit für Kinder und solcher für Pflegebedürftige. Für nach 1992 geborene Kinder werden den Eltern (in der Regel der Mutter) je drei Versicherungsjahre auch additiv zu einer Erwerbsarbeit angerechnet, für früher geborene Kinder inzwischen zweieinhalb Jahre („Mütterrente“, Anhebung von einem auf zwei Jahre seit Juli 2014 und auf 2,5 Jahre seit 2019), bewertet mit dem Durchschnittseinkommen aller Versicherten. Zudem profitieren Mütter in den sogenannten Kinderberücksichtigungszeiten (bis zum vollendeten 10. Lebensjahr des Kindes) zusätzlich bei Ausübung einer Teilzeitarbeit oder einer unterdurchschnittlich bezahlten Tätigkeit von einer Aufwertung ihrer Anwartschaften. Im Vergleich dazu werden Pflegetätigkeiten in wesentlich geringerem Umfang in der GRV berücksichtigt. Seit Einführung der Pflegeversicherung 1995 wird die nicht-professionelle häusliche Pflege zwar besser als zuvor rentenrechtlich abgebildet. Gemäß $\mathbb{3}$ Abs. 1 Nr.1a SGB VI besteht für nicht erwerbsmäßig tätige Pflegepersonen eine Versicherungspflicht in der GRV. Dabei richten sich die Beiträge nach dem pflegerischen und zeitlichen Aufwand 
der Pflegepersonen. Mit dem Inkrafttreten des Pflegestärkungsgesetzes II (PSG II) im Januar 2017 wurde der Rentenanspruch, der aus Pflegearbeit erwachsen kann, erhöht. 2018 lag er bei ganzjähriger Pflegearbeit je nach Pflegegrad, Pflegeleistung und Region zwischen 5,57 Euro und 30,90 Euro pro Monat (Deutsche Rentenversicherung Bund 2020). Die Zahlung von Renten-Pflichtbeiträgen für pflegende Angehörige durch die Pflegekassen ist allerdings an zahlreiche Voraussetzungen gebunden. ${ }^{3}$ So werden keine Beiträge für Personen gezahlt, die mehr als 30 Stunden erwerbstätig sind anders als im Fall der Sorge für Kinder während der ersten drei Jahre. Angesichts der im Vergleich zur Kindererziehung geringeren Honorierung von Pflegetätigkeiten wäre zu prüfen, wie die Anrechnung von Pflegezeiten so verbessert werden kann, dass eine gleiche gesellschaftliche Wertschätzung für beide Arten von Sorgetätigkeiten zum Ausdruck kommt. Dies schließt eine stärker additive Anrechnung von Pflegetätigkeiten bei Kombination mit Erwerbstätigkeit und/oder Rentenbezug ein. Zudem ist die Beschränkung auf die Pflege naher Angehörige zu überdenken; private, nicht erwerbsmäßige Pflege sollte auch dann zur Erhöhung von Rentenanwartschaften führen, wenn nicht verwandte Personen, z. B. Nachbarn oder Freunde, gepflegt werden.

Sobald eine Pflegeperson das Rentenalter erreicht hat, wird die Zahlung der Pflichtbeiträge eingestellt. Dies ist problematisch, denn Pflegetätigkeiten verteilen sich anders über den Lebensverlauf als Erwerbstätigkeit; gerade Frauen pflegen häufig auch nach Erreichen des Rentenalters. Daher sollte die Anrechnung im Zuge einer allgemeinen Flexibilisierung des Rentenübergangs, zu dem schon erste Schritte durch das Flexirentengesetz von 2016 gegangen worden waren (Bundesregierung 2016), auch auf Pflegende höheren Alters ausgedehnt werden. Anzustreben wäre, dass auch nach dem Erreichen des gesetzlichen Rentenalters und dem Beginn des Rentenbezugs weitere Rentenansprüche für Pflege (und Erwerbstätigkeit) erworben werden können.

3 Ab 2017 gelten nach PSG II folgende Bedingungen, damit Pflege rentenerhöhend wirkt: Der/die Pflegebedürftige muss einen Pflegegrad von 2 bis 5 haben; die Pflege muss in häuslicher Umgebung stattfinden; sie muss mindestens zehn Stunden pro Woche verteilt auf mindestens zwei Tage in Anspruch nehmen. 


\subsubsection{Anwartschaftssplitting bei (Ehe-)Paaren}

Seit 2002 können Ehepaare statt einer Hinterbliebenenrente aus der GRV ein sogenanntes Rentensplitting wählen. Haben beide Eheleute jeweils mindestens 25 Jahre mit rentenrechtlichen Zeiten zurückgelegt, können so die während der Ehezeit erworbenen Rentenanwartschaften partnerschaftlich aufgeteilt werden - vergleichbar mit dem Versorgungsausgleich bei einer Ehescheidung. Im Unterschied zum Versorgungsausgleich werden in das Rentensplitting allerdings nur GRV-Anwartschaften einbezogen, keine Anwartschaften aus der zweiten oder dritten Säule. Seit 2005 gibt es die Splittingoption auch für eingetragene Lebenspartnerschaften. Paare, die das Rentensplitting wählen, müssen dies in einer gemeinschaftlichen Erklärung festlegen. Dem Optionsmodell zufolge wird das Rentensplitting in folgenden Fällen durchgeführt: wenn beide erstmals Anspruch auf eine Altersvollrente aus der GRV haben; wenn erstmalig ein Partner oder eine Partnerin einen Altersvollrentenanspruch und der andere die Regelaltersgrenze erreicht hat; wenn ein Partner oder eine Partnerin verstirbt, bevor diese Voraussetzungen vorliegen. Im Gegenzug entfallen abgeleitete Hinterbliebenenrenten $(\mathbb{S} 120 \mathrm{a}$ SGB VI). Die Möglichkeit eines Splittings der Anwartschaften hat bisher als freiwillige Option jedoch kaum Wirkung entfaltet. Aus Gleichstellungsperspektive scheint das Anwartschaftssplitting jedoch gegenüber der abgeleiteten Absicherung über Hinterbliebenenrenten zeitgemäß, da es mit dem Aufbau eigenständiger Rentenansprüche korrespondiert. Unabhängig von der innerfamiliären Arbeitsteilung bauen hier beide Partner in gleichem Ausmaß Rentenanwartschaften auf. Zwar führt die Teilung der Anwartschaften nicht zu einem höheren Haushaltseinkommen im Alter und bei Verwitwung liegt das Versorgungsniveau der überlebenden Person oft niedriger als bei heutiger Berechnung. Jedoch bleiben wie im Scheidungsfall die Anwartschaften erhalten, die sich aus der Übertragung von Entgeltpunkten ergeben: Ein auf dem Wege des Rentensplittings erworbener eigenständiger Rentenanspruch entfällt bei Wiederheirat oder bei Vorliegen von eigenem (Erwerbs-)Einkommen nicht. Die Sachverständigenkommission für den Zweiten Gleichstellungsbericht hat daher empfohlen, ein permanentes Anwartschaftssplitting für in Ehe und eingetragener Lebenspartnerschaft erworbene Ansprüche als Regelmodell einzuführen. Dies korrespondiert mit der Empfehlung, die Errungenschaftsgemeinschaft als gesetzlichen Güterstand in Ehe und eingetragener Lebenspartnerschaft vorzusehen. In das Anwartschaftssplitting sollten längerfristig auch Betriebsrentenansprüche sowie Ansprüche aus berufsständischen Versorgungswerken und der Beamtenversorgung einbezogen werden. Angesichts der Verteilungswirkungen sind allerdings Ver- 
trauensschutz- und Übergangsregelungen zu berücksichtigen, so dass die Einführung des Splittings als Regelmodell auf neue Ehen und eingetragene Lebenspartnerschaften begrenzt werden sollte. Ein entsprechendes Anwartschafts- und Rentensplitting ist ein anspruchsvolles Reformprojekt, bei dem verschiedene Punkte zu klären sind, wie die Frage, ob alle Ansprüche gesplittet oder nur ein Grundbetrag übertragen werden soll, und ob permanent, beim Eintreten des ersten Rentenfalls oder erst beim Rentenzugang des zweiten Partners gesplittet werden soll. Insbesondere wäre zu berücksichtigen und auch zu kommunizieren, dass zwischen den Renteneintritten des ersten und des zweiten Partners und nach dem Tod des ersten Partners das Renteneinkommen des Ehepaar-Haushalts niedriger liegen kann als bisher - analog zur schon bestehenden Situation im Scheidungsfall. Die Schweiz kann als Beispiel für ein Land genannt werden, in dem eine entsprechende Übertragung von Rentenanwartschaften zwischen (Ehe-)Partnern seit langem offenbar erfolgreich praktiziert und akzeptiert wird.

\subsection{Wie kann das Sicherungsversprechen für langjährig GRV-Versicherte eingelöst werden?}

Die Frage, wie die Situation von GRV-Versicherten verbessert werden kann, die trotz langjähriger Versicherungsbiografie und Beitragszahlung aufgrund ihres unterdurchschnittlichem Einkommen keine Alterssicherungsansprüche oberhalb der Grundsicherung erreichen, war in den vergangenen Jahren wiederholt Thema der politischen Debatte und Gegenstand unterschiedlicher Reformvorschläge. Die folgenden Ausführungen konzentrieren sich auf das zum Zeitpunkt der Erstellung dieses Beitrags im Stadium des Referentenentwurfs vorliegende, aktuelle „Gesetz zur Einführung der Grundrente für langjährig in der gesetzlichen Rentenversicherung Versicherte mit unterdurchschnittlichem Einkommen und für weitere Maßnahmen zur Erhöhung der Alterseinkommen“ (Grundrentengesetz - GruReG, Fassung vom 16.01.2020). 


\subsubsection{Das aktuelle Konzept der Grundrente}

Dem Referentenentwurf für das GruReG zufolge (BMAS 2020) sind „zur Stärkung des Grundversprechens des Sozialstaates und der Leistungsfähigkeit der gesetzlichen Rentenversicherung insgesamt, insbesondere aber für langjährig Versicherte mit unterdurchschnittlichem Einkommen, [...] folgende Maßnahmen vorgesehen:

1. Die Einführung einer Grundrente für langjährig Versicherte in der gesetzlichen Rentenversicherung und

2. die Einführung von Freibeträgen im Wohngeld, in der Grundsicherung für Arbeitsuchende (SGB II), in der Hilfe zum Lebensunterhalt, in der Grundsicherung im Alter und bei Erwerbsminderung (SGB XII) und in den fürsorgerischen Leistungen der Sozialen Entschädigung.

Herzstück ist die Grundrente für langjährig Versicherte mit unterdurchschnittlichem Einkommen. Sie ist als Rentenzuschlag konzipiert und soll von einer nachzuweisenden Bedürftigkeit wie in den Fürsorgesystemen unabhängig sein" (BMAS 2020, S. 1f.). Anspruch auf Grundrente soll haben, wer mindestens 33 Jahre „Grundrentenzeiten“ aufweisen kann, „das sind vor allem Zeiten, in denen Pflichtbeiträge aufgrund einer Beschäftigung, Kindererziehung oder Pflegetätigkeit an die gesetzliche Rentenversicherung gezahlt wurden“. In diesem Fall „soll die Rente um einen ,Zuschlag' erhöht werden, wenn die Entgeltpunkte des Erwerbslebens unterdurchschnittlich, aber nicht ganz gering waren“. Hiermit soll sichergestellt werden, dass langjährig Pflichtversicherte und auch Fürsorgeleistende im Alter mehr erhalten als die Grundsicherung nach SGB XII, denn: „Die Menschen müssen darauf vertrauen können, dass sie nach einem langen Arbeitsleben - auch bei unterdurchschnittlichem Einkommen - ordentlich abgesichert sind und besser dastehen als jemand, der wenig oder gar nicht gearbeitet und somit wenige oder keine Pflichtbeiträge zur gesetzlichen Rentenversicherung gezahlt hat" (BMAS 2020, S. 1f.).

Die vorgesehenen Anspruchsvoraussetzungen und Regelungen zur Aufwertung zeichnen sich durch eine beträchtliche Komplexität aus (vgl. Sozialbeirat 2019). An dieser Stelle soll es aber nicht um die Regelungen im Einzelnen gehen. Genau so wenig steht im Folgenden die Frage im Fokus, ob die geplante Grundrente tatsächlich ein adäquates Instrument ist, um der „Lebensleistung“ Fürsorge leistender Frauen gerecht zu werden (zu einer kritischen Einschätzung s. Deutscher Juristinnenbund 2020). Stattdessen geht es um das mit den Grundsicherungsplänen verbundene Ziel, 
„das Vertrauen in das Grundversprechen des Sozialstaats und in die Leistungsfähigkeit der gesetzlichen Rentenversicherung zu stärken" (BMAS 2020, S. 1).

\subsubsection{Grundrente als beitragsfinanzierte Versicherungsleistung?!}

Zwei miteinander verknüpfte Fragen liegen auf der Hand: Wie wäre eine entsprechende Leistung systematisch einzuordnen - und wie wäre sie ggf. zu finanzieren? Der Ruf nach Steuerfinanzierung vereint bei der Einführung entsprechender neuer Leistungen regelmäßig Arbeitgeberseite und Gewerkschaften im gemeinsamen Ziel, einen weiteren Anstieg der Beitragssätze zu verhindern. Er ist noch nachdrücklicher geworden, seit die Mütterrente eingeführt wurde und aus den Beiträgen der Versicherten finanziert wird. Doch der Ruf nach Steuerfinanzierung ist regelmäßig auch von jenen zu hören, die die Systematik der Gesetzlichen Rentenversicherung in Gefahr sehen. Sofern "gesamtgesellschaftliche“ Leistungen nicht aus Steuern, sondern aus Beiträgen finanziert werden, führe dies, so die Argumentation, zu einer "Verwässerung“ des Konzepts der einkommensbezogenen und beitragsfinanzierten gesetzlichen Rentenversicherung. Dies verringere die Rendite der eingezahlten Beiträge - auch im Vergleich zu Formen privater Absicherung - und könne die Akzeptanz der GRV bei den Versicherten schmälern (so z.B. Schmähl 2019, Ruland 2019).

Allerdings fehlt es ungeachtet einiger vorliegender Arbeiten (als früher Überblick: Kube 2002) bis heute an einer unumstrittenen Definition, welche Leistungen als „versicherungsfremd“, „gesamtgesellschaftlich“ oder „nicht beitragsgedeckt“ zu bewerten ist. Dies hat der Sozialbeirat in seinem Gutachten von 2019 als Problem markiert: „Der Sozialbeirat sieht es als unbefriedigend an, dass die Frage einer sachgerechten Aufteilung der Finanzierung der Rentenversicherung in Steuer- und Beitragsanteile bis heute ungelöst ist. [...] So gehen die Meinungen darüber auseinander, wie Leistungen wie etwa die Grundrente in dieser Hinsicht systematisch einzuordnen sind. Der Sozialbeirat hält es daher für geboten, die Frage der sachgerechten Finanzierung der Rentenversicherung nach Beitrags- und Steueranteilen grundsätzlich zu klären und nicht weiter diskretionär zu beantworten" (Sozialbeirat 2019, Ziffer 51).

Im Folgenden wird argumentiert, dass Überlegungen zu einem definierten Mindestsicherungsziel für langjährig Versicherte sinnvoll und keineswegs systemfremd für eine gesetzliche Rentenversicherung der ersten Säule sind, und dass insofern eine Beitragsfinanzierung für die geplante Grund- 
rente sachgerecht wäre. ${ }^{4}$ Dies stellt nicht infrage, dass gesamtgesellschaftliche Aufgaben über Steuern von der Allgemeinheit getragen werden sollten - zumindest solange, wie nur ein eingeschränkter Ausschnitt der Bevölkerung in der GRV versichert ist und Beiträge entrichtet. So wäre es systematisch richtig, Leistungen wie Kindererziehungszeiten respektive Mütterrenten, die der ganzen Bevölkerung offenstehen und niedrigschwellig auch bei Personen ohne weitere GRV-Versicherungszeiten zu Leistungsansprüchen führen, ${ }^{5}$ über Steuern zu finanzieren. Hingegen ist die Grundrente bewusst auf langjährig rentenversicherte Personen ausgerichtet und damit eine exklusive Leistung für einen besonders eng und dauerhaft an die GRV gebundenen Personenkreis. Angesichts der selektiven Zugangsvoraussetzungen zur GRV (erinnert sei an Selbständige, geringfügig Beschäftigte, erst recht Nichterwerbstätige) kann hier nicht von einer Leistung für die gesamte Gesellschaft gesprochen werden. Zwar gibt es gute Gründe dafür, das Risiko unzureichender Alterseinkünfte als einen Tatbestand aufzufassen, der eine „gesamtgesellschaftliche“ Aufgabe darstellt und politische Interventionen rechtfertigt. Dann müsste eine Leistung zur Verminderung der Altersarmut jedoch auch anderen Gruppen als langjährig GRV-Versicherten zugutekommen. Dass andererseits beitragsfinanzierte Umverteilung innerhalb der Versichertengemeinschaft der Rentenversicherung inhärent ist, zeigt insbesondere diejenige zwischen Gesunden und Erwerbsgeminderten, zwischen Personen unterschiedlicher sozialer Klassen aufgrund ihrer unterschiedlichen Lebenserwartung (Brussig/Schulz 2019) oder zwischen Alleinstehenden und Ehepaaren durch die beitragsfinanzierte Hinterbliebenenversorgung (schon Schmähl 1998, Rürup 2004). Diese Umverteilung - auch als Risikoausgleich bezeichnet - ist also keineswegs systemfremd im Rahmen einer Sozialversicherung. Dass die Ausgestaltung der Hinterbliebenenrente strittig ist, ändert nichts an der Tatsache, dass diese Leistung nicht nur existiert, sondern offenbar auch verfassungsgemäß ist. Bei der Grundrente handelt es also sich um eine Leistung, die exklusiv auf eine bestimmte Versichertengruppe beschränkt ist. „Armutsvermeidung“ - als gesamtgesellschaftliche Aufgabe - ist zwar für einen Teil der Berechtigten gewollte Nebenfolge. Doch sie steht nicht im Vordergrund. Vielmehr kann die Leistung als Versicherung gegen das Risiko, trotz langjähriger Mitgliedschaft und Beitragszahlung keine armutsvermeiden-

4 Der folgende Teil stützt sich auf einen mit Gert Wagner veröffentlichten Artikel der Verfasserin (Klammer/Wagner 2020).

5 So reichen zwei Kinder (6 Entgeltpunkte) zur Begründung eines - wenn auch niedrigen - Rentenanspruchs in der GRV. 
den Alterssicherungsansprüche aufbauen zu können betrachtet werden. Dies ist - neben der Versicherung gegen Langlebigkeit, gegen Erwerbsminderung und gegen den Tod mit Hinterlassung Hinterbliebener - ein viertes Risiko, das die GRV zukünftig für die Gruppe der Versicherten abdecken kann und sollte und insoweit keineswegs eine „versicherungsfremde Leistung“. Wie ausgeführt, ist durch die 2001 eingeleitete Absenkung des Rentenniveaus das Risiko, trotz langjähriger Systemzugehörigkeit und Beitragszahlung keinen Rentenanspruch oberhalb des Grundsicherungsniveaus zu erreichen, zunehmend gestiegen und wird weiter steigen. Angesichts der Kombination aus der Absenkung des Rentenniveaus mit der $\mathrm{Zu}$ nahme von Teilzeitarbeit und Niedriglöhnen kann das Sicherungsversprechen der GRV, vor Armut im Alter zu schützen, für einen steigenden Teil der Versicherten nicht mehr eingelöst werden. Es ist daher die Frage zu stellen, ob die Legitimität und Akzeptanz der Rentenversicherung nicht stärker dadurch gefährdet ist, dass Menschen ihr Leben lang arbeiten, ordnungsgemäß einzahlen und dennoch nicht mehr erhalten als andere, die nie Beiträge entrichtet haben, als es der Fall wäre, wenn innerhalb der Solidargemeinschaft GRV bei Wahrung der Teilhabeäquivalenz eine relative Besserstellung von Personen mit schwachen Erwerbsbiografien zulasten besser verdienender Beitragszahler stattfinden würde. Ungleiche Leistungshöhen innerhalb der Rentenversicherung tragen dazu bei, „dass die Beitragslast von den Betroffenen akzeptiert wird“ (Wallrabenstein 2019, S. 312), allerdings kommt es „in keiner Weise auf eine mathematisch exakte Äquivalenz der Leistung im Verhältnis zur Beitragshöhe an“ (ebd.). Diese lässt sich angesichts vieler relevanter Variablen, zumal im Lebensverlauf, ohnehin kaum objektiv feststellen. Relevant ist vielmehr das Gefühl der Fairness sozialstaatlicher Leistungen - im Vergleich zu anderen Versicherten, aber auch im Vergleich zu Nichtversicherten.

Betrachtet man das Risiko, trotz langjähriger Erwerbstätigkeit keine armutsvermeidenden Rentenansprüche aufbauen zu können, als ein Risiko, das ex ante jeden Versicherten treffen kann, so stellt seine Absicherung keine Umverteilung, sondern einen Risikoausgleich zwischen Versicherten dar, der für viele staatliche Rentensysteme der ersten Säule in Europa und darüber hinaus durchaus konstitutiv ist. ${ }^{6}$ Durch die Grundrente könnte grundsätzlich - auch wenn sie in einzelnen Punkten und angesichts ihrer Komplexität sicherlich kritisierbar ist - die Legitimation der Rentenversicherung in der Bevölkerung gestärkt werden, insofern bestimmte Folgen

6 Genannt seien bspw. die Rentensysteme der Schweiz, Österreichs oder der Niederlande, wenn sie auch auf unterschiedlichen Finanzierungsregelungen beruhen. 
der Deregulierung des Arbeitsmarktes, die zu niedrigen Löhnen und unstetigeren Erwerbsverläufen führen, das Sicherungsversprechen der GRV in Frage stellen (so auch Köhler-Rama 2019, S. 433ff.). Die Verlässlichkeit ist zudem bei einem beitragsfinanzierten Risikoausgleich eher gesichert als bei einer steuerfinanzierten Leistung, da die hieraus erwachsenden Ansprüche besser geschützt sind. Beiträge in die GRV, die zur Milderung der sozialen Folgen der Deregulierung beitragen, entlasten zugleich bei den Steuern, da angesichts des grundgesetzlich verbrieften Sozialstaatsgebots ansonsten über Steuern zusätzliche Grundsicherungsleistungen zu finanzieren wären.

\section{Risiken für unzureichende Alterseinkommen ursachengerecht angehen- breite Versicherungspflicht einführen!}

Sowohl die hier diskutierten Vorschläge zur Verbesserung der Alterssicherung von Frauen unter Anerkennung ihrer „Lebensleistung“ als auch der aktuelle Plan des Bundesarbeitsministers zur Einführung einer Grundrente für langjährig GRV-Versicherte „reparieren“ allerdings letztlich die Folgen eines unzureichenden Erwerbs an Altersrentenansprüchen da, wo das Kind schon in den Brunnen gefallen ist: am Ende der Erwerbsbiografie. Für eine vorausschauende, vorbeugende Alterssicherungspolitik muss jedoch - das kann nicht oft genug wiederholt werden - die Ursachenbekämpfung früher beginnen: Bei der Gestaltung guter Arbeitsbedingungen und Löhnen, die daran orientiert sind, dass Menschen bis zum Renteneintritt erwerbstätig sein können. Löhne müssen zudem nicht nur zur Bestreitung des gegenwärtigen Lebensunterhalts ausreichen, sondern zugleich auch einen angemessenen Vorsorgebeitrag für eine im Durchschnitt heute rund 20jährige Rentenphase abwerfen. Im Sinne eines nachhaltigen Rentensystems, das eine Überbelastung zukünftiger Generationen und (Steuer-)Zahler vermeidet, wäre auch - wie anderweitig ausführlich ausgeführt (z.B. Brettschneider/Klammer 2016, S. 408-416) - eine umfassende Erweiterung der Versicherungspflicht auf andere Gruppen und Einkommensarten entlang des Lebenslaufs zielführend. Mit der von der Bundesregierung geplanten Versicherungspflicht für Selbständige soll hier eine erste Lücke geschlossen werden. Niedrige (Stunden-)Löhne sollten durch die Einführung einer Mindestbemessungsgrundlage für Rentenbeiträge auf Arbeitsentgelt (Steffen 2014) kompensiert werden. Für das Ziel, Männern wie Frauen den Aufbau armutsvermeidender eigenständiger Rentenansprüche zu ermöglichen und unerwünschte Verteilungswirkungen zu reduzieren, sollte die GRV als zentrale Säule der deutschen Alterssicherung gestärkt und zu 
einer universellen Versicherung mit Mindestsicherungsziel ausgebaut werden. Die Sorge vor unzureichenden Rentenansprüchen zu verkleinern, gehört zu einer vernünftigen Gesellschaftspolitik, die nicht nur den Beschäftigten, sondern auch den Arbeitgebern nutzt. Eine durchgängige Versicherungspflicht kann dazu beitragen, nicht nachhaltige Erwerbsverhältnisse aufzudecken, die bisher akzeptiert werden, da von ihnen kein Beitrag zur Alterssicherung zu entrichten ist und daher ihre langfristigen individuellen und gesellschaftlichen Folgen nicht sichtbar werden. So hilft eine durchgängige Versicherungspflicht, Defizite beim Aufbau armutsvermeidender Alterssicherungsansprüche dort zu identifizieren, wo sie entstehen, und die gefühlte „Gerechtigkeitslücke“ zu verringern, die dadurch entstanden ist, dass zunehmend Personen mit langen Beitragszeiten und solche, die keine oder nur wenige Beiträge eingezahlt haben, im Alter gleichermaBen auf die Grundsicherung im Alter verwiesen werden.

\section{Literatur}

Adriaans, J./Eisnecker, P./Liebig, S. (2019): Gerechtigkeit im europäischen Vergleich: Verteilung nach Bedarf und Leistung in Deutschland besonders befürwortet, in: DIW Wochenbericht 45/2019, S. 817-825

Blank, F./Blum, S. (2017): Kindererziehungszeiten in der Alterssicherung. Ein Vergleich sechs europäischer Länder, WSI Working Paper Nr. 209, Düsseldorf

BMAS - Bundesministerium für Arbeit und Soziales (2019): Rentenversicherungsbericht 2019, Berlin

BMAS (2020): Entwurf eines Gesetzes zur Einführung der Grundrente für langjährig in der gesetzlichen Rentenversicherung Versicherte mit unterdurchschnittlichem Einkommen und für weitere Maßnahmen zur Erhöhung der Alterseinkommen" (Grundrentengesetz - GruReG), Fassung vom 16.01.2020, Berlin

Brettschneider, A./Klammer, U. (2016): Lebenswege in die Altersarmut. Biografische Analysen und sozialpolitische Perspektiven, Berlin

Brussig, M./Klammer, U./Langer, P. (2019): Einordnung gesellschaftspolitischer Debatten zur sozialen Ungleichheit, BMAS-Forschungsbericht 537, Berlin

Brussig, M./Schulz, S. E. (2019): Soziale Unterschiede im Mortalitätsrisiko, Das frühere Arbeitsleben beeinflusst die fernere Lebenserwartung, IAQ-Report 6/2019, https://www.iaq.uni-due.de/iaq-report/2019/report2019-06.pdf (abgerufen am 20.01.2020)

Bundesregierung (2011): Neue Wege - Gleiche Chancen, Gleichstellung von Frauen und Männern im Lebensverlauf. Erster Gleichstellungsbericht, BundestagsDrucksache 17/6240, Berlin 
Bundesregierung (2016): Gesetz zur Flexibilisierung des Übergangs vom Erwerbsleben in den Ruhestand und zur Stärkung von Rehabilitation und Prävention im Erwerbsleben (Flexirentengesetz) vom 8.12.2016, Bundesgesetzblatt 2016 Tl. I Nr. 59, 13.12.2016, Bonn.

Bundesregierung (2017): Zweiter Gleichstellungsbericht der Bundesregierung, Bundestags-Drucksache 18/12840 vom 21.06.2017, Berlin

Buslei, H./Geyer, J./Haan, P./Harnisch, M. (2019): Starke Nichtinanspruchnahme von Grundsicherung deutet auf hohe verdeckte Altersarmut, in: DIW Wochenbericht 49/2019, S. 909-917

Deutsche Rentenversicherung Bund (2020): Pflege von Angehörigen lohnt sich auch für die Rente, https://www.deutsche-rentenversicherung.de/DRV/DE/Rent e/Familie-und-Kinder/Angehoerige-pflegen/angehoerige-pflegen_node.html (abgerufen am 24.01.2020)

Deutscher Juristinnenbund (2020): Stellungnahme zum Entwurf eines Gesetzes zur Einführung der Grundrente für langjährig in der gesetzlichen Rentenversicherung Versicherte mit unterdurchschnittlichem Einkommen und für weitere Maßnahmen zur Erhöhung der Alterseinkommen (Stand: 16.01.2020), 20.01.2020, Berlin

Eisnecker, P./Adriaans, J./Liebig, S. (2018): Was macht Gerechtigkeit aus? Deutsche WählerInnen befürworten über Parteigrenzen hinweg das Leistungs- und das Bedarfsprinzip, DIW aktuell 17/2018, Berlin

Götte, S. (2015): Wahrnehmung von Armut und Reichtum in Deutschland. Ergebnisse der repräsentativen Bevölkerungsbefragung „ARB Survey 2015“, Bonn

Klammer, U. (2016): Alterssicherung von Frauen revisited - aktuelle Entwicklungen und künftige Perspektiven, in: Sozialer Fortschritt 66 (5), S. 359-375

Klammer, U./Wagner, G. G. (2020): Grundrentenplan der großen Koalition. Welche Risiken soll die gesetzliche Rentenversicherung versichern? In: Wirtschaftsdienst 100 (1), S. 29-34

Kumpmann, I./Gühne, M./Buscher, H. (2010): Armut im Alter - Ursachenanalyse und eine Projektion für das Jahr 2023, IHW Diskussionspapier 8, Halle

Köhler-Rama, T. (2019): Grundrente ist kein Systembruch - eine Replik, in: Wirtschaftsdienst 99 (6), S. 432-436

Kube, H. (2002): Äquivalenz und Solidarität im Sozialversicherungsrecht, in: Der Staat 41 (3), S. 452-478

Rürup, B. (2004): Vom Bundeszuschuss zum Bundesbeitrag in der gesetzlichen Rentenversicherung, in: Deutsche Rentenversicherung 10/2004, S. 586-591

Ruland, F. (2019): Vorschlag zur Grundrente: ungerecht, ineffizient und teuer, in: Wirtschaftsdienst 99 (3), S. 189-195

Schmähl, W. (1998): Finanzpolitik und Rentenversicherung - Beispiele für die Notwendigkeit einer integrierten Sichtweise, in: Galler, H. P./Wagner, G. G. (Hrsg.): Empirische Forschung und wirtschaftspolitische Beratung - Festschrift für Hans-Jürgen Krupp zum 65. Geburtstag, Frankfurt/New York, S. 448-468

Schmähl, W. (2019): „Grundrente“: Koalitionskitt mit Folgen, in: Wirtschaftsdienst 99 (12), S. 2-3 
Sozialbeirat (2019): Gutachten des Sozialbeirats zum Rentenversicherungsbericht 2019, Berlin

Sozialpolitik-aktuell.de (2020): Empfängerquoten von Grundsicherung im Alter und bei Erwerbsminderung 2003-2018 in \% der jeweiligen Bevölkerungsgruppe, am Jahresende, http://www.sozialpolitik-aktuell.de/tl_files/sozialpolitik-aktu ell/_Politikfelder/Sozialstaat/Datensammlung/PDF-Dateien/abbIII51.pdf (abgerufen am 13.02.2020)

Statista (2018): Dossier „Armut in Deutschland“, https://de.statista.com/statistik/stu die/id/50108/dokument/armut-in-deutschland/ (abgerufen am 15.11.2018)

Steffen, J. (2014): Wenn der Mindestlohn fürs Alter nicht reicht. Plädoyer für eine Mindestbemessungsgrundlage für Rentenbeiträge auf Arbeitsentgelt, http://ww w.portal-sozialpolitik.de/uploads/sopo/pdf/2014/2014-01-00 \%20Mindestbemess ungsgrundlage_PS.pdf (abgerufen am 11.08.2016)

Wallrabenstein, A. (2019): Äquivalenz in der Sozialversicherung - aber am richtigen Ort, in: Durner, W./Reimer, F./Spieker, I./Wallrabenstein, A. (Hrsg.): Das sinnvoll Denkbare denken, das davon Machbare machen. Gedächtnisschrift für Arndt Schmehl, Berlin, S. 303-315 